\title{
Analytical calculation of axial optical force on a Rayleigh particle illuminated by Gaussian beams beyond the paraxial approximation
}

\author{
Jun Chen, ${ }^{1, *}$ Jack Ng, ${ }^{2}$ Shiyang Liu, ${ }^{1}$ and Zhifang Lin $^{1}$ \\ ${ }^{1}$ Surface Physics Laboratory, Department of Physics, Fudan University, Shanghai 200433, China \\ ${ }^{2}$ Department of Physics, The Hong Kong University of Science and Technology, Kowloon, Hong Kong \\ (Received 1 April 2009; revised manuscript received 3 June 2009; published 27 August 2009)
}

\begin{abstract}
We investigate the optical trapping of a Rayleigh particle by a linearly or radially polarized Gaussian beam. The Mie theory is applied to obtain a full solution, with the incident beam being described by the mixed dipole model, which is beyond the paraxial approximation. We then obtain approximate analytical expressions for the optical force, equilibrium position, and trap stiffness for a Rayleigh particle. At equilibrium, the displacement for the particle from the focus scales like $a^{3}$ (where $a$ is the radius) for a transparent particle, owing to scattering, whereas for absorptive particles it scales like $C+D a^{2}$, owing to absorption. The trap stiffness is found to be proportional to $a^{3}$, in agreement with the recent experiment. The radially polarized beam is found to be superior to the linearly polarized beam in the Rayleigh regime in terms of its ability to trap. It is found that the larger the ratio of $\varepsilon_{r} / \varepsilon_{i}$, the closer the equilibrium to the focus, and thus higher stability.
\end{abstract}

DOI: 10.1103/PhysRevE.80.026607

PACS number(s): 42.50.Wk, 87.80.Cc, 42.25.Fx, 42.62.-b

\section{INTRODUCTION}

Optical tweezers [1-18], introduced by Ashkin et al. in 1986, has been developed into a powerful and versatile experimental tool. It has found application in the frontier of a variety of exciting research fields, such as atomic physics, colloidal science, nanoscience, and biology [16,19-22]. While optical tweezer is such a versatile and convenient experimental tool, the theoretical modeling of optical tweezers is by no means an easy task. Quantitative agreement between the experiment and the theoretical modeling was achieved only in recent years $[15,17,18]$. Moreover, these accurate theoretical works rely on heavy numerical simulation, consequently the underlying physics is not transparent. The difficulty in modeling optical tweezers resides in the fact that the trapping beam is a tightly focused beam, where the conventional paraxial description of laser beam fails. In this paper, the Mie theory is applied to calculate the optical force, with the trapping beam being modeled by the mixed dipole model theory [23]. In this mixed dipole model theory, the field singularities are replaced by phase singularities, thus it can be physically realized [24]. The resultant beam is a rigorous solution to the Maxwell equations, furthermore, the model proves efficient in modeling Gaussian beam near the focal region beyond the paraxial limit [25]. Thus our results go beyond the conventional paraxial approximation. Moreover, in the limit of a loosely focused beam, our result reduces to that of the paraxial approximation by Harada [8]. We then develop an analytical model for an optically trapped Rayleigh particle ( $a / \lambda \ll 1$, where $a$ is the radius). It is found that the equilibrium position of an optically trapped Rayleigh particle is slightly displaced away from the focus and biased toward the propagating direction of the beam, and this bias is qualitatively different for absorptive and transparent particles. This bias goes like $a^{3}$ for transparent particles, owing

\footnotetext{
*Author to whom correspondence should be addressed; 071019010@fudan.edu.cn
}

to scattering, and equals to $C+D a^{2}$ for absorptive particles, owing to absorption. Here $C$ and $D$ are constants independent of $a$. An expression for the trap stiffness is derived, which is found to be proportional to $a^{3}$, in agreement with the recent experimental measurements of Hansen et al. [26]. This is not entirely unexpected since the gradient force is responsible for the trap stiffness and the gradient force is proportional to $a^{3}$. The explicit analytical expressions of the scattering force and the gradient force are also derived. It was previously demonstrated by Kawauchi [27] and Nieminen [28] that radially polarized beams can be used to improve the performance of optical tweezers in the geometrical optics regime and the Mie regime, owing to the reduced scattering. Here we shall show that the radially polarized beams are also superior to the linearly polarized beams in the Rayleigh regime.

The paper is organized as follows. In Sec. II, some fundamental formulas for calculating forces on a spherical particle are derived using the Mie theory, with the incident Gaussian beam being described by the mixed dipole model. Simplified analytical expressions of the forces are presented in Sec. III, for both absorptive and transparent particles. Differences between our forces expressions and those by Harada are tabulated in Appendix B. In Sec. IV, the analytical approach as well as the Mie theory approach are applied to study the properties of optically trapped particle. The dependency of the equilibrium positions for both absorptive and transparent particles on the permittivities is investigated for both linearly and radially polarized Gaussian beams. The superiority of radially polarized Gaussian beam over linearly polarized beam is clearly demonstrated in trapping a Rayleigh particle. Section V is the conclusion. Details on some results in force expressions are tabulated in the appendixes.

\section{GENERAL FORMULATION}

In this section, using the mixed dipole model to describe the incident trapping beam, we present the formalism for the calculations of optical forces exerted on a spherical particle. 
We consider a spherical particle with permittivity $\varepsilon_{0} \varepsilon$ surrounded by a homogeneous and lossless medium with permittivity $\varepsilon_{0}$. Since we are working in optical frequency, most materials are nonmagnetic. Accordingly, we take $\mu=\mu_{0}$ for both the particle and its background medium.

\section{A. Optical force calculated by Mie theory}

We choose the origin of our coordinate system to be located at the center of the sphere. According to the Mie theory [29-32], the incident electromagnetic fields $\left(\boldsymbol{E}_{\text {inc }}, \boldsymbol{H}_{\text {inc }}\right)$, the scattered fields $\left(\boldsymbol{E}_{s c a}, \boldsymbol{H}_{s c a}\right)$ and the internal fields $\left(\boldsymbol{E}_{\text {int }}, \boldsymbol{H}_{\text {int }}\right)$ can be expanded in terms of the vector spherical wave functions $\left(\boldsymbol{M}_{m n}^{(1)}, \boldsymbol{N}_{m n}^{(1)}\right)$ and $\left(\boldsymbol{M}_{m n}^{(3)}, \boldsymbol{N}_{m n}^{(3)}\right)[33-37]$,

$$
\begin{gathered}
\boldsymbol{E}_{i n c}=-\sum_{n=1}^{\infty} \sum_{m=-n}^{n}\left[\bar{p}_{m n} \boldsymbol{N}_{m n}^{(1)}(k \boldsymbol{r})+\bar{q}_{m n} \boldsymbol{M}_{m n}^{(1)}(k \boldsymbol{r})\right], \\
\boldsymbol{E}_{s c a}=\sum_{n=1}^{\infty} \sum_{m=-n}^{n}\left[\bar{a}_{m n} \boldsymbol{N}_{m n}^{(3)}(k \boldsymbol{r})+\bar{b}_{m n} \boldsymbol{M}_{m n}^{(3)}(k \boldsymbol{r})\right], \\
\boldsymbol{E}_{\text {int }}=-\sum_{n=1}^{\infty} \sum_{m=-n}^{n}\left[\bar{d}_{m n} \boldsymbol{N}_{m n}^{(1)}\left(k_{s} \boldsymbol{r}\right)+\bar{c}_{m n} \boldsymbol{M}_{m n}^{(1)}\left(k_{s} \boldsymbol{r}\right)\right], \\
\boldsymbol{H}_{i n c}=\frac{i k}{\omega \mu_{0}} \sum_{n=1}^{\infty} \sum_{m=-n}^{n}\left[\bar{q}_{m n} \boldsymbol{N}_{m n}^{(1)}(k \boldsymbol{r})+\bar{p}_{m n} \boldsymbol{M}_{m n}^{(1)}(k \boldsymbol{r})\right], \\
\boldsymbol{H}_{s c a}=-\frac{i k}{\omega \mu_{0}} \sum_{n=1}^{\infty} \sum_{m=-n}^{n}\left[\bar{b}_{m n} \boldsymbol{N}_{m n}^{(3)}(k \boldsymbol{r})+\bar{a}_{m n} \boldsymbol{M}_{m n}^{(3)}(k \boldsymbol{r})\right], \\
\boldsymbol{H}_{i n t}=\frac{i k_{s}}{\omega \mu_{0}} \sum_{n=1}^{\infty} \sum_{m=-n}^{n}\left[\bar{c}_{m n} \boldsymbol{N}_{m n}^{(1)}\left(k_{s} \boldsymbol{r}\right)+\bar{d}_{m n} \boldsymbol{M}_{m n}^{(1)}\left(k_{s} \boldsymbol{r}\right)\right],
\end{gathered}
$$

where

$$
\begin{gathered}
\bar{p}_{m n}=i E_{m n} p_{m n}, \quad \bar{q}_{m n}=i E_{m n} q_{m n}, \\
\bar{a}_{m n}=i E_{m n} a_{m n}, \quad \bar{b}_{m n}=i E_{m n} b_{m n}, \\
\bar{d}_{m n}=i E_{m n} d_{m n}, \quad \bar{c}_{m n}=i E_{m n} c_{m n}, \\
E_{m n}=\left|E_{0}\right| i^{n}\left[\frac{(2 n+1)(n-m) !}{n(n+1)(n+m) !}\right]^{1 / 2},
\end{gathered}
$$

$k_{s}(k)$ denotes the wave number inside the sphere (surrounding medium). Here the superscript 1 and 3 in the vector spherical harmonics denote its radial function, with 1 corresponds to the spherical Bessel function $j_{n}$ and 3 corresponds to the spherical Hankel function of the first kind $h_{n}^{(1)}$. For the incident fields (1a) and (2a) and the internal fields (1c) and (2c), the spherical Bessel function is employed since the field must be finite at the origin. For the scattered fields (1b) and (2b), since they are outgoing waves, the spherical Hankel function of the first kind is employed. by

The expansion coefficients of the incident fields are given

$$
\begin{aligned}
& p_{m n}=\frac{k r}{j_{n}(k r)} \oiint_{\Omega}\left[\boldsymbol{e}_{r} \cdot \boldsymbol{E}_{i n c}(r, \theta, \phi)\right] \mathcal{F}_{n, m}(\theta, \phi) d \Omega, \\
& q_{m n}=\frac{Z k r}{j_{n}(k r)} \oiint_{\Omega}\left[\boldsymbol{e}_{r} \cdot \boldsymbol{H}_{i n c}(r, \theta, \phi)\right] \mathcal{F}_{n, m}(\theta, \phi) d \Omega,
\end{aligned}
$$

where $Z=\sqrt{\frac{\mu_{0}}{\varepsilon_{0}}}$ is the impedance and the function $\mathcal{F}_{n, m}$ in the integrand is defined as

$$
\mathcal{F}_{n, m}(\theta, \phi)=\frac{i^{1-n}}{\sqrt{4 \pi n(n+1)}\left|E_{0}\right|} Y_{n, m}^{*}(\theta, \phi),
$$

with $Y_{n, m}^{*}(\theta, \phi)$ denoting the complex conjugate of the spherical harmonic function

$$
Y_{n, m}^{*}(\theta, \phi)=\left[\frac{(2 n+1)}{4 \pi} \frac{(n-m) !}{(n+m) !}\right]^{1 / 2} P_{n}^{m}(\cos \theta) e^{-i m \phi} .
$$

Here, we define two dimensionless parameters where

$$
\rho=k a, \quad m_{s}=\frac{k_{s}}{k},
$$

where $a$ denotes the radius of the sphere.

The total fields outside the sphere are

$$
\boldsymbol{E}_{\text {ext }}=\boldsymbol{E}_{\text {sca }}+\boldsymbol{E}_{\text {inc }}, \quad \boldsymbol{H}_{\text {ext }}=\boldsymbol{H}_{\text {sca }}+\boldsymbol{H}_{\text {inc }} .
$$

By applying the standard boundary conditions

$$
\left(\boldsymbol{E}_{\text {ext }}-\boldsymbol{E}_{\text {int }}\right) \times \boldsymbol{e}_{r}=0, \quad\left(\boldsymbol{H}_{\text {ext }}-\boldsymbol{H}_{\text {int }}\right) \times \boldsymbol{e}_{r}=0,
$$

we can relate the expansion coefficients of the scattered fields to that of the incident fields,

$$
a_{m n}=a_{n} p_{m n}, \quad b_{m n}=b_{n} q_{m n},
$$

where the Mie coefficients $a_{n}$ and $b_{n}$ are given by

$$
\begin{aligned}
& a_{n}=\frac{m_{s} \psi_{n}\left(m_{s} \rho\right) \psi_{n}^{\prime}(\rho)-\psi_{n}(\rho) \psi_{n}^{\prime}\left(m_{s} \rho\right)}{m_{s} \psi_{n}\left(m_{s} \rho\right) \xi_{n}^{\prime}(\rho)-\xi_{n}(\rho) \psi_{n}^{\prime}\left(m_{s} \rho\right)}, \\
& b_{n}=\frac{\psi_{n}\left(m_{s} \rho\right) \psi_{n}^{\prime}(\rho)-m_{s} \psi_{n}(\rho) \psi_{n}^{\prime}\left(m_{s} \rho\right)}{\psi_{n}\left(m_{s} \rho\right) \xi_{n}^{\prime}(\rho)-m_{s} \xi_{n}(\rho) \psi_{n}^{\prime}\left(m_{s} \rho\right)},
\end{aligned}
$$

and the Ricatti-Bessel functions $\psi_{n}, \xi_{n}$ are given by [34]

$$
\psi_{n}(\rho)=\rho j_{n}(\rho), \quad \xi_{n}(\rho)=\rho h_{n}^{(1)}(\rho) .
$$

where $j_{n}(\rho)$ and $h_{n}^{(1)}(\rho)$ are, respectively, the spherical Bessel function and the spherical Hankel function of the first kind.

Outside the particle, the time-averaged Maxwell stress tensor can be obtained from the incident and the scattered fields,

$$
\boldsymbol{T}=\frac{1}{2} \operatorname{Re}\left[\boldsymbol{E}_{\text {ext }} \boldsymbol{D}_{\text {ext }}^{*}-\frac{1}{2} \boldsymbol{E}_{\text {ext }} \cdot \boldsymbol{D}_{\text {ext }}^{*} \boldsymbol{I}+\boldsymbol{H}_{\text {ext }} \boldsymbol{B}_{\text {ext }}^{*}-\frac{1}{2} \boldsymbol{H}_{\text {ext }} \cdot \boldsymbol{B}_{\text {ext }}^{*} \boldsymbol{I}\right] .
$$

By integrating the Maxwell stress tensor over a closed surface, the time-averaged force can be obtained [38,39], 


$$
\boldsymbol{F}=\oiint d \boldsymbol{S} \cdot \boldsymbol{T}=\oiint\left[\boldsymbol{e}_{r} \cdot \boldsymbol{T}\right] d \sigma=\oiint\left[\boldsymbol{T} \cdot \boldsymbol{e}_{r}\right] d \sigma,
$$

we can derive the three Cartesian components of optical forces as $[40,41]$

$$
F_{x}=\operatorname{Re}\left[F_{1}\right], \quad F_{y}=\operatorname{Im}\left[F_{1}\right], \quad F_{z}=\operatorname{Re}\left[F_{2}\right],
$$

where

$$
\begin{aligned}
F_{1}= & \frac{2 \pi \varepsilon_{0}}{k^{2}}\left|E_{0}\right|^{2} \sum_{n=1}^{\infty} \sum_{m=-n}^{n}\left\{\frac{[(n-m)(n+m+1)]^{1 / 2}}{n(n+1)}\right. \\
& \times\left(\widetilde{a}_{m, n} \widetilde{b}_{m+1, n}^{*}+\widetilde{b}_{m, n} \widetilde{a}_{m+1, n}^{*}-\widetilde{p}_{m, n} \widetilde{q}_{m+1, n}^{*}-\widetilde{q}_{m, n} \widetilde{p}_{m+1, n}^{*}\right) \\
& -\left[\frac{n(n+2)(n+m+1)(n+m+2)}{(n+1)^{2}(2 n+1)(2 n+3)}\right]^{1 / 2} \\
& \times\left(\widetilde{a}_{m, n} \widetilde{a}_{m+1, n+1}^{*}+\widetilde{b}_{m, n} \widetilde{b}_{m+1, n+1}^{*}-\widetilde{p}_{m, n} \widetilde{p}_{m+1, n+1}^{*}\right. \\
& \left.-\widetilde{q}_{m, n} \widetilde{q}_{m+1, n+1}^{*}\right)+\left[\frac{n(n+2)(n-m)(n-m+1)}{(n+1)^{2}(2 n+1)(2 n+3)}\right]^{1 / 2} \\
& \times\left(\widetilde{a}_{m, n+1} \widetilde{a}_{m+1, n}^{*}+\widetilde{b}_{m, n+1} \widetilde{b}_{m+1, n}^{*}-\widetilde{p}_{m, n+1} \widetilde{p}_{m+1, n}^{*}\right. \\
& \left.\left.-\widetilde{q}_{m, n+1} \widetilde{q}_{m+1, n}^{*}\right)\right\}, \\
F_{2}= & -\frac{4 \pi \varepsilon_{0}}{k^{2}}\left|E_{0}\right|^{2} \sum_{n=1}^{\infty} \sum_{m=-n}^{n}\left\{\frac{m}{n(n+1)} \times\left(\widetilde{a}_{m, n} \widetilde{b}_{m, n}^{*}-\widetilde{p}_{m, n} \widetilde{q}_{m, n}^{*}\right)\right. \\
& +\left[\frac{n(n+2)(n-m+1)(n+m+1)}{(n+1)^{2}(2 n+1)(2 n+3)}\right]^{1 / 2} \\
& \left.\times\left(\widetilde{a}_{m, n} \widetilde{a}_{m, n+1}^{*}+\widetilde{b}_{m, n} \widetilde{b}_{m, n+1}^{*}-\widetilde{p}_{m, n} \widetilde{p}_{m, n+1}^{*}-\widetilde{q}_{m, n} \widetilde{q}_{m, n+1}^{*}\right)\right\},
\end{aligned}
$$

and

$$
\begin{aligned}
& \tilde{a}_{m, n}=a_{m, n}-\frac{1}{2} p_{m, n}, \quad \widetilde{p}_{m, n}=\frac{1}{2} p_{m, n}, \\
& \tilde{b}_{m, n}=b_{m, n}-\frac{1}{2} q_{m, n}, \quad \widetilde{q}_{m, n}=\frac{1}{2} q_{m, n} .
\end{aligned}
$$

We note that up to now, we have not yet specified the expansion coefficients of the incident fields. Consequently the expressions of the forces Eq. (12) are general and applicable to an arbitrary incident field. In the following sections, we shall apply this formalism to a specific problem: a particle illuminated by a Gaussian beam described by the mixed dipole model. We shall also study the problem in the long wavelength limit, in which case, simple analytical expressions of the optical forces can be obtained.

\section{B. Mixed dipole model for linearly and radially polarized Gaussian beam}

Traditionally, the Gaussian beam is treated in the paraxial limit, nevertheless, such paraxial beam is not a rigorous so- lution to Maxwell equations, and thus it is not physically realizable. Although the problem is not serious for a loosely focused laser beam, it becomes significant for a tightly focused beam, as in the case of optical tweezers. In order to overcome this difficulty, instead of the paraxial Gaussian beam, we take the mixed dipole model (complex sources and sinks model) to describe the incident Gaussian beam, which is first introduced by Sheppard and Saghafi [23]. The resultant beam is a rigorous solution to the Maxwell equations, moreover, the model proves efficient in modeling Gaussian beam near the focal region beyond the paraxial limit [25]. We use this model to describe the field near the focus of a Gaussian beam. The region near the focus is the region that we are most interested. We note that this model has already been used in many works $[25,42,43]$.

In this model, in order to avoid nonphysical singularities in the focal plane at a radius equal to the confocal parameter, for a linearly polarized Gaussian beam, Sheppard [23] suggested that the vector potential of the dipoles be written as

$$
\boldsymbol{A}_{p}=\frac{\boldsymbol{A}_{p x}+\boldsymbol{A}_{p x}^{*}}{2 i}, \quad \boldsymbol{A}_{m}=\frac{\boldsymbol{A}_{m y}+\boldsymbol{A}_{m y}^{*}}{2 i},
$$

where

$$
\boldsymbol{A}_{p x}=\frac{e^{i k R}}{i k R} \boldsymbol{e}_{x}, \quad \boldsymbol{A}_{m y}=\frac{e^{i k R}}{i k R} \boldsymbol{e}_{y},
$$

are, respectively, the vector potential of an electric dipole $\boldsymbol{p}$ $=p_{0} \boldsymbol{e}_{x}$ and a magnetic dipole $\boldsymbol{m}=m_{0} \boldsymbol{e}_{y}$ located at a virtual location of $\left(0,0, z_{0}+i l_{0}\right)$, where $\left(0,0, z_{0}\right)$ is the center of the trapping beam, $l_{0}=\frac{1}{2} k w_{0}^{2}$ is the Rayleigh diffraction length, and $w_{0}$ is the waist radius. Here $p_{0}$ and $m_{0}$ are the amplitude of $\boldsymbol{p}$ and $\boldsymbol{m}$, respectively, and we have set $p_{0}=1$ and $m_{0}=1$ for convenience. Accordingly the trapping beam, which propagates along $+z$ direction, can be expressed as

$$
\begin{aligned}
\boldsymbol{E}= & E_{0} k\left(l_{0}-i z_{0}\right) \exp \left(-k l_{0}\right) \\
& \times\left[\frac{i}{k^{2}} \boldsymbol{\nabla} \times\left(\boldsymbol{\nabla} \times \boldsymbol{A}_{p}\right)-\frac{1}{k} \boldsymbol{\nabla} \times \boldsymbol{A}_{m}\right], \\
\boldsymbol{H}= & Z^{-1} E_{0} k\left(l_{0}-i z_{0}\right) \exp \left(-k l_{0}\right) \\
& \times\left[\frac{i}{k^{2}} \boldsymbol{\nabla} \times\left(\boldsymbol{\nabla} \times \boldsymbol{A}_{m}\right)+\frac{1}{k} \boldsymbol{\nabla} \times \boldsymbol{A}_{p}\right] .
\end{aligned}
$$

By substituting Eq. (14) into Eq. (16), it is straightforward to find the radial components of incident electromagnetic fields $\boldsymbol{e}_{r} \cdot \boldsymbol{E}_{\text {inc }}(r, \theta, \phi)$ and $\boldsymbol{e}_{r} \cdot \boldsymbol{H}_{i n c}(r, \theta, \phi)$. By inserting these expressions into Eq. (4), explicit expressions for $p_{m n}$ and $q_{m n}$ for a beam propagating along $z$ axis are obtained,

$$
\begin{gathered}
p_{1 n}=\frac{\sqrt{2 n+1}}{2 i^{n}}\left[\frac{(n+1) i}{2 n+1} j_{n-1}\left(i k z_{c}\right)+j_{n}\left(i k z_{c}\right)\right. \\
\left.-\frac{n i}{2 n+1} j_{n+1}\left(i k z_{c}\right)\right] \frac{k l_{0}}{e^{k l_{0}}}, \\
p_{1 n}=-p_{-1 n}=q_{1 n}=q_{-1 n},
\end{gathered}
$$




$$
p_{m n}=q_{m n}=0, \text { if } m \neq \pm 1,
$$

with $z_{c}=l_{0}-i z_{0}$.

In addition, for a sphere in a radially polarized Gaussian beam, only the vector potential of an electric dipole $\boldsymbol{p}=p_{0} \boldsymbol{e}_{z}$ needs to be considered, and it is given by $\boldsymbol{A}_{p}=\frac{\sin k R}{i k R} \boldsymbol{e}_{z}$, and the corresponding $p_{m n}$ and $q_{m n}$ are

$$
\begin{gathered}
p_{0 n}=\frac{l_{0}}{i^{n} z_{c}} \sqrt{(2 n+1) n(n+1)} j_{n}\left(i k z_{c}\right) e^{-k l_{0}}, \\
q_{0 n}=0, \quad p_{m n}=q_{m n}=0, \quad \text { if } \quad m \neq 0 .
\end{gathered}
$$

\section{ANALYTICAL CALCULATIONS}

Based on the theory outlined in the preceding parts of the paper, this section presents some analytical results in the long wavelength limit. We shall only present the calculation details for a particle trapped by a linearly polarized Gaussian beam, and the case for a radially polarized Gaussian beam can be treated likewise. Throughout this paper, the linearly polarized Gaussian beam is polarized along the $x$ direction.

Based on Eqs. (13a), (13b), and (17), we can derive, in the long wavelength limit, a simple analytical expression of the optical force. It should be noted that the gradient force dominates along the transverse directions (i.e., $x$ and $y$ directions), so particles with positive polarizability (such as dielectric particles) are always stable along the transverse directions, as they are attracted by the intensity maximum. Likewise, particles with negative polarizability (such as metallic particles) are always unstable along the transverse directions, as they are repelled by the intensity maximum. Consequently, we shall only concentrate on the longitudinal force $F_{z}$ for particle with positive polarizability. For particles with positive polarizability, whether or not a particle can be trapped depends on whether the gradient force along the $z$ direction is strong enough to overcome the scattering force.

By inserting Eqs. (13c) and (17) into Eq. (13b), we obtain the Taylor series expansion of $F_{z}$,

$$
F_{z}=\operatorname{Re}\left[F_{2}\right]=c_{3} \rho^{3}+c_{5} \rho^{5}+c_{6} \rho^{6}+o\left(\rho^{7}\right),
$$

where terms that are higher than $\rho^{6}$ are truncated. Although we have already expanded $F_{z}$ in a Taylor series of $\rho$, the expansion coefficients $c_{3}, c_{5}$, and $c_{6}$ are still complicated and further approximations are needed. We thus simplify Eq. (19) based on the following principles:

(i) We neglect terms that are associated with $e^{-2 k l_{0}}, e^{-4 k l_{0}}$, and so on. This can be well justified. Consider for example, a focused Gaussian beam with the waist radius $w_{0}=\lambda(\lambda$ is the wavelength), $k l_{0}=2 \pi^{2}$, then $e^{-2 k l_{0}} \sim 7 \times 10^{-18}, e^{-4 k l_{0}} \sim 5$ $\times 10^{-35}$, which are negligibly small.

(ii) The particle is close to the center of the beam, i.e., $\left|z_{0}\right| \ll l_{0}$. This is again reasonable, since this is the region where optical trapping happens.

By introducing two dimensionless parameters $\vartheta=k l_{0}$ and $\varrho=k z_{0}$, we have the relationship

$$
|\varrho| \ll \vartheta
$$

\section{A. Transparent particle}

For a transparent particle, the permittivity $\varepsilon$ of the sphere is real. In order to facilitate the understanding of the physics associated with the individual terms in the force expressions, we compare our formulas with those of the plane wave case. Force on a sphere induced by a plane wave can be entirely attributed to the radiation pressure or scattering force

$$
F_{z}^{p w}=\frac{4 \pi \varepsilon_{0} E_{0}^{2}}{k^{2}} \frac{(\varepsilon-1)^{2}}{3(\varepsilon+2)^{2}} \rho^{6} .
$$

The corresponding term in the case of a linearly polarized Gaussian beam is

$$
F_{z, s c a}^{G b}=c_{6} \rho^{6}=\frac{4 \pi \varepsilon_{0} E_{0}^{2}}{k^{2}} \frac{(\varepsilon-1)^{2}}{3(\varepsilon+2)^{2}} g_{0} \rho^{6},
$$

where $g_{0}$ is given in Appendix A.

Using Eq. (20) in Eq. (22), Eq. (22) can be simplified to

$$
F_{z, s c a}^{G b}=\frac{4 \pi \varepsilon_{0} E_{0}^{2}}{k^{2}} \frac{(\varepsilon-1)^{2}}{3(\varepsilon+2)^{2}} g(\vartheta) \rho^{6},
$$

which is independent of the location of the sphere and differs from Eq. (21) only by a factor $g(\vartheta)$ defined as

$$
g(\vartheta)=1-\frac{3}{\vartheta}+\frac{5}{\vartheta^{2}}-\frac{5}{\vartheta^{3}}+\frac{11}{4 \vartheta^{4}}-\frac{3}{4 \vartheta^{5}} .
$$

Note that the factor $g(\vartheta)$ depends only on the characteristic parameter of the Gaussian beam $\vartheta=k l_{0}$, and in the limit $l_{0}$ $\rightarrow \infty$ where a linearly polarized Gaussian beam turns into a plane wave, $g(\vartheta) \rightarrow 1$, thus $F_{z, s c a}^{G b}$ carries the same physical meaning as $F_{z}^{p w}$.

For a sphere illuminated by a Gaussian beam, there is also a gradient force due to the inhomogeneity of the field intensity,

$$
F_{z, \text { grad }}=c_{3} \rho^{3}+c_{5} \rho^{5},
$$

where

$$
\begin{gathered}
c_{3}=\frac{2 \pi \varepsilon_{0} E_{0}^{2}}{k^{2}} \frac{(\varepsilon-1) \varrho}{(\varepsilon+2) \vartheta^{2}} h_{0}, \\
c_{5}=\frac{2 \pi \varepsilon_{0} E_{0}^{2}}{k^{2}} \frac{\varrho}{60(\varepsilon+2)^{2}(2 \varepsilon+3) \vartheta^{8}} h_{1},
\end{gathered}
$$

and $h_{0}$ and $h_{1}$ are given in Appendix A.

Using Eq. (20) and retaining only the dominating $\rho^{3}$ term in Eq. (25), Eq. (25) can be simplified to

$$
F_{z, \text { grad }}=\frac{2 \pi \varepsilon_{0} E_{0}^{2}}{k^{2}} \frac{(\varepsilon-1) \varrho}{(\varepsilon+2) \vartheta^{2}} h(\vartheta) \rho^{3},
$$

where

$$
h(\vartheta)=1-\frac{4}{\vartheta}+\frac{6}{\vartheta^{2}}-\frac{3}{\vartheta^{3}}+\frac{3}{4 \vartheta^{4}} .
$$

Note that $F_{z \text {,grad }}$ vanishes as $l_{0} \rightarrow \infty$, which corresponds to the situation of a plane wave. 
To provide a lucid explanation of the physical meaning of Eq. (27), we calculate the gradient force acting on a dipolar particle with polarizability [44]

$$
\alpha=4 \pi \varepsilon_{0} a^{3}\left(\frac{\varepsilon-1}{\varepsilon+2}\right) .
$$

From Eqs. (14) and (16), it is straightforward to show that Eq. (27) equals to

$$
F_{z, \text { grad }}=\left.\frac{\alpha}{4} \nabla\left(|\boldsymbol{E}|^{2}\right)\right|_{x=y=0} .
$$

i.e., Eq. (27) is the gradient force.

In the conventional paraxial approximation, the paraxial Gaussian beam in fact satisfies the approximate paraxial wave equation, rather than the Maxwell equations. In our theory, by employing the mixed dipole model (the resultant beam is a rigorous solution to the Maxwell equations, furthermore, the model proves efficient in modeling Gaussian beam near the focal region beyond paraxial limit [23,25]), our force expressions are results beyond paraxial approximation. We also tabulated the differences between our force expressions and those of the paraxial approximation [8] in Appendix B. We note that these differences vanish when $\vartheta$ $=k l_{0}=\frac{1}{2} k^{2} w_{0}^{2}$ is large, which precisely corresponds to the paraxial limit.

A particle will be trapped at the position where the scattering force and the gradient force balance each other,

$$
\left.\left(F_{z, s c a}+F_{z, g r a d}\right)\right|_{z_{0}=z_{e q u}}=0 .
$$

From Eqs. (23), (27), and (31), the equilibrium position is given by

$$
z_{\text {equ }}=-\frac{2(\varepsilon-1)}{3 k(\varepsilon+2)} \frac{g(\vartheta)}{h(\vartheta)} \vartheta^{2} \rho^{3},
$$

Note that $z_{\text {equ }}$ in Eq. (32) is the position for the center of the beam, whereas the particle is located at the origin. In the case of a loosely focused Gaussian beam (for example, the waist radius $w_{0}=\lambda$, and therefore $\left.\vartheta=2 \pi^{2}\right), \frac{g(\vartheta)}{h(\vartheta)} \approx 1$, the equilibrium position can be simplified into

$$
z_{e q u}=-\frac{(\varepsilon-1) k^{6}}{6(\varepsilon+2)} a^{3} w_{0}^{4} .
$$

From Eq. (33), we can see the $a^{3}$ and $w_{0}^{4}$ dependence of the equilibrium position. It is clear from Eq. (33) that the larger the particle, the further away the equilibrium from the beam center (owing to scattering force), and the smaller the beam waist, the closer the equilibrium from the beam center (owing to stronger gradient force).

\section{B. Absorptive particle}

For an absorptive particle, its refractive index is a complex number. There appears another force component owing to absorption in the scattering force expression for this case whereas it disappears for the transparent case, and we will see later that this absorptive force will dominates in the scattering force. In order to distinguish the different meanings between the absorptive force component and the original scattering force, we denote the absorptive term as $F_{z, a b s}$, and denote the original scattering component still as $F_{z, s c a}$. The forces acting on a sphere that is illuminated by a plane wave are given by

$$
F_{z, s c a}^{p w}=\frac{4 \pi \varepsilon_{0} E_{0}^{2}}{3 k^{2}} q\left(n_{R}, n_{I}\right) \rho^{6},
$$

$$
F_{z, a b s}^{p w}=\frac{4 \pi \varepsilon_{0} E_{0}^{2}}{k^{2}} u\left(n_{R}, n_{I}\right) \rho^{3}+\frac{2 \pi \varepsilon_{0} E_{0}^{2}}{15 k^{2}} p\left(n_{R}, n_{I}\right) \rho^{5},
$$

where $q\left(n_{R}, n_{I}\right), u\left(n_{R}, n_{I}\right)$, and $p\left(n_{R}, n_{I}\right)$ are tabulated in Appendix $\mathrm{A}$, and $n_{R}$ and $n_{I}$ are the real and imaginary part of the complex refractive index, respectively. On the other hand, for an absorptive particle illuminated by a linearly polarized Gaussian beam, the forces are

$$
F_{z}=F_{z, s c a}+F_{z, g r a d}+F_{z, a b s},
$$

with

$$
\begin{gathered}
F_{z, s c a}=c_{6}^{e} \rho^{6}, \\
F_{z, g r a d}=c_{3}^{o} \rho^{3}+c_{5}^{o} \rho^{5}, \\
F_{z, a b s}=c_{3}^{e} \rho^{3}+c_{5}^{e} \rho^{5}+c_{6}^{o} \rho^{6},
\end{gathered}
$$

Here $c_{i}^{e}$ and $c_{i}^{o}$ are, respectively, the even and odd series in the Taylor series expansion of $c_{i}$ in terms of $\eta=\frac{z_{0}}{l_{0}}$ at $\eta=0$. Similar to Eq. (30), Eq. (36b) can also be written as the gradient of the field intensity

$$
F_{z, \text { grad }}=\frac{1}{4} \operatorname{Re}\left[\left.\alpha \boldsymbol{\nabla}\left(|\boldsymbol{E}|^{2}\right)\right|_{x=y=0}\right] .
$$

Using Eq. (20) and keep only the leading order terms $\eta^{0}$, $\eta^{1}$ in $c_{3}, c_{5}$, and $c_{6}$, Eq. (36) becomes

$$
\begin{gathered}
F_{z, s c a}=\zeta_{6}^{0} \rho^{6}, \\
F_{z, \text { grad }}=\zeta_{3}^{1} \varrho \rho^{3}+\zeta_{5}^{1} \varrho \rho^{5}, \\
F_{z, a b s}=\zeta_{3}^{0} \rho^{3}+\zeta_{5}^{0} \rho^{5}+\zeta_{6}^{1} \varrho \rho^{6},
\end{gathered}
$$

where $\zeta_{i}^{0}$ and $\zeta_{i}^{1}$ are the coefficients of $\eta^{0}$ and $\eta^{1}$ in the series expansion of $c_{i}$ respectively. By further truncating terms that are of the order of $\rho^{4}$ or higher in Eqs. (38b) and (38c), the force expressions are simplified into

$$
\begin{gathered}
F_{z, s c a}=\frac{4 \pi \varepsilon_{0} E_{0}^{2}}{3 k^{2}} q\left(n_{R}, n_{I}\right) g(\vartheta) \rho^{6}, \\
F_{z, \text { grad }}=\frac{2 \pi \varepsilon_{0} E_{0}^{2}}{k^{2}} v\left(n_{R}, n_{I}\right) h(\vartheta) \vartheta^{-2} \varrho \rho^{3},
\end{gathered}
$$




$$
F_{z, a b s}=\frac{4 \pi \varepsilon_{0} E_{0}^{2}}{k^{2}} u\left(n_{R}, n_{I}\right) g(\vartheta) \rho^{3},
$$

where $g(\vartheta)$ and $h(\vartheta)$ are the same as Eqs. (24) and (28), $q\left(n_{R}, n_{I}\right)$ and $u\left(n_{R}, n_{I}\right)$ are the same as those defined in Eq. (34), and $v\left(n_{R}, n_{I}\right)$ is given in Appendix A.

The particle will be trapped at an equilibrium position whenever

$$
\left.\left(F_{z, s c a}+F_{z, a b s}+F_{z, g r a d}\right)\right|_{z_{0}=z_{e q u}}=0 .
$$

By inserting Eq. (38) into Eq. (40), the equilibrium position for beam center is given by

$$
z_{\text {equ }}=-\frac{\zeta_{3}^{0}}{\zeta_{3}^{1}}-\left[\frac{\zeta_{5}^{0}}{\zeta_{3}^{1}}-\frac{\zeta_{3}^{0} \zeta_{5}^{1}}{\left(\zeta_{3}^{1}\right)^{2}}\right] \rho^{2}-\left[\frac{\zeta_{6}^{0}}{\zeta_{3}^{1}}-\frac{\zeta_{3}^{0} \zeta_{6}^{1}}{\left(\zeta_{3}^{1}\right)^{2}}\right] \rho^{3} .
$$

It is noted that the $a^{3}$ dependence of equilibrium position in Eq. (33) for a transparent particle is no longer hold for an absorptive particle. Accordingly, the size scalings of the equilibrium positions are qualitatively different for absorptive and transparent particles. For absorptive particle, owing to the absorptive force, the equilibrium position is given by $C+D a^{2}$, where $C$ and $D$ are constant.

As mentioned above, the force acting on a particle located near the focus of the beam can be expressed in the form of $F_{z}=-\kappa_{z}\left(z_{\text {equ }}-z_{0}\right)$, where $\kappa_{z}$ is defined as stiffness and $z_{0}$ is the coordinate of the focus. From Eqs. (35), (39b), and (39c), we have

$$
\kappa_{z}=2 \pi \varepsilon_{0} E_{0}^{2} \frac{2-n_{R}^{2}+n_{I}^{2}-\left(n_{R}^{2}+n_{I}^{2}\right)^{2}}{4\left(1+n_{R}^{2}-n_{I}^{2}\right)+\left(n_{R}^{2}+n_{I}^{2}\right)^{2}} \frac{a^{3}}{l_{0}^{2}} h(\vartheta),
$$

which indicates the $a^{3}$ scaling in the long wavelength limit. We note that the $a^{3}$ scaling of the trap stiffness in Eq. (42) is valid for both absorptive and transparent particles. Moreover, it agrees with recent experiments [26].

\section{APPLICATION}

In this section, we apply the formalism we developed earlier to explore the physics of optical trapping. Equation (19) is valid only if (i) $|\varepsilon|$ is not too large and (ii) $\varepsilon$ is not in the neighborhood of the plasmon resonance of $\varepsilon_{c}=-2$. In order to study how the equilibrium position varies with $\varepsilon$, the equilibrium position as a function of $\varepsilon$ is plotted in Fig. 1. In Fig. 1 , the white region denotes that no equilibrium position exists. The bottom level of the color bar in Fig. 1 corresponds to sphere whose equilibrium position is close to the center of the Gaussian beam, whereas the top level corresponds to the maximal equilibrium position. It is clear that compared to a linearly polarized Gaussian beam, particles illuminated by a radially polarized Gaussian beam are better trapped (much nearer to the center of the beam), and it has a much wider stable region in terms of $\varepsilon_{r}, \varepsilon_{i}$, and $\rho$ (where $\varepsilon_{r}$ and $\varepsilon_{i}$ are the real and imaginary parts of the complex permittivity, respectively, and $\rho=k a$ ), for which a particle can be trapped. We can see that, equilibrium positions for different dielectric materials are rather different. The larger the ratio of $\varepsilon_{r} / \varepsilon_{i}$, the closer the particle to the focus, and thus higher stability.

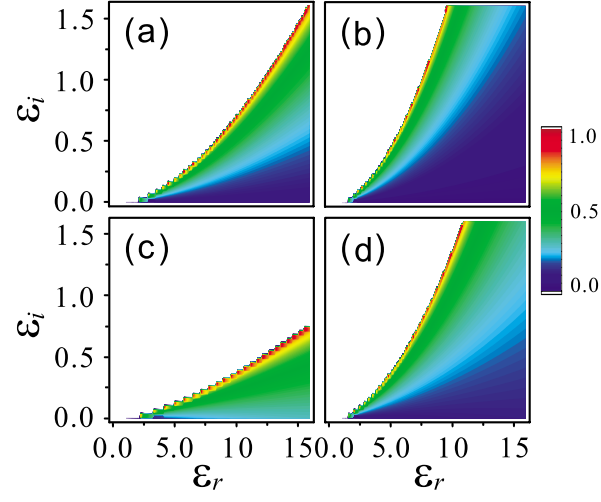

FIG. 1. (Color online) Equilibrium position as a function of complex permittivity for a sphere illuminated by a Gaussian beam. The waist radius $w_{0}=\lambda$. The size parameter $\rho=0.2$ for (a) and (b), and $\rho=0.3$ for (c) and (d). (a) and (c) are for the linearly polarized case (x polarized), (b) and (d) are for the radially polarized case.

In Fig. 2, we compare our analytical results in the Rayleigh limit directly with that of Harada [8], as well as that of the accurate numerical calculation based on the Mie theory, for a polystyrene sphere in water $\left[\varepsilon=(1.592 / 1.332)^{2}\right]$, and as a function of the normalized axial position of $z / k w_{0}^{2}$. In the Mie theory calculation, Eq. (13b) is truncated at $n=2$, which is sufficient for the small spheres $(k a<1.6)$ that we consider in this paper. In the small particle regime, especially for the weakly focused Gaussian beam, the three approaches agree very well with each others, as shown in Fig. 2(a). However, for a strongly focused Gaussian beam $\left(w_{0}=0.25 \mu \mathrm{m}\right)$, the approach of Harada deviates from the others, as shown in Fig. 2(c). It is clear that our results agree with the exact results more than those of Harada. Our analytical results deviate from the numerical results in Figs. 2(b) and 2(d). This is due to the fact that $\rho=1.6>1$, which is beyond the Rayleigh regime and therefore the small particle assumption in our analytical results does not hold anymore. We also find that, for a weakly focused Gaussian beam with the waist radius $w_{0}=2.2 \mu \mathrm{m}$, we cannot trap particles with $\rho>0.2$, and for a strongly focused Gaussian beam with the waist

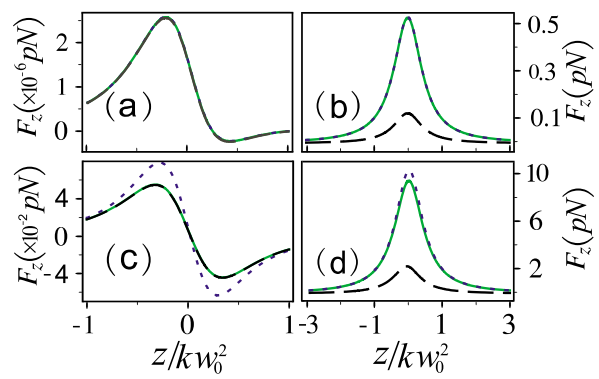

FIG. 2. (Color online) Axial optical force versus $z / k w_{0}^{2}$ for a polystyrene sphere in water $\left[\varepsilon=(1.592 / 1.332)^{2}\right]$. The dashed black curves are calculated by the accurate Mie theory, the solid green curves are calculated by our analytical approach, and the dotted blue curves are calculated by the approach of Harada. The wavelength of Gaussian beam $\lambda=514.5 \mathrm{~nm}$ and beam power $P$ $=100 \mathrm{~mW}$. (a) $w_{0}=2.2 \mu \mathrm{m}, \rho=0.2$ and (b) $w_{0}=2.2 \mu \mathrm{m}, \rho=1.6$, (c) $w_{0}=0.25 \mu \mathrm{m}, \rho=0.4$ and (d) $w_{0}=0.5 \mu \mathrm{m}, \rho=1.6$. 


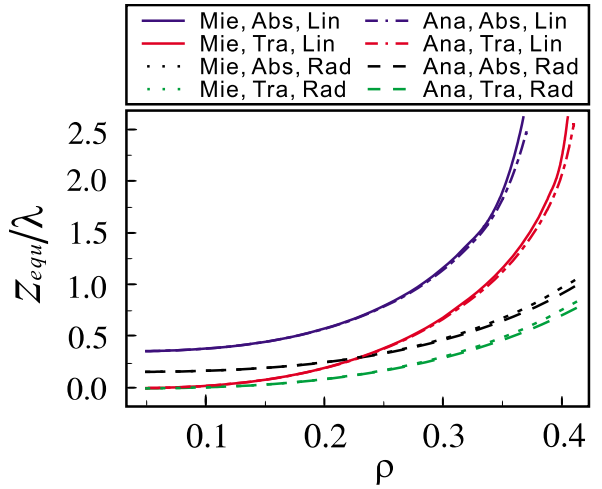

FIG. 3. (Color online) Comparison for $z_{\text {equ }} / \lambda$ versus $\rho$ between simplified analytical results and the accurate Mie theory. A spherical nanoparticle in air is illuminated either by a radially polarized or a linearly polarized Gaussian beam with the wavelength $\lambda$ $=1064 \mathrm{~nm}$ and the waist radius $w_{0}=\lambda$. Both the transparent particle with $\varepsilon=5$ and the absorptive particle with $\varepsilon=5+0.05 i$ are considered. In the figure legend, Mie=Mie Theory, Ana=Analytical, Abs $=$ Absorption, Tra $=$ Transparent, Lin $=$ Linear Polarization, Rad $=$ Radial Polarization.

radius $w_{0}=0.25 \mu \mathrm{m}$, we cannot trap particles with $\rho>0.9$ (data not shown). Accordingly, the higher degree of focusing of a Gaussian beam, the bigger particles we can trap.

The accuracy of our small particle approximation in predicting the equilibrium position of a trapped small particle is illustrated in Fig. 3. One can see that for a particle with small size parameter $\rho$, our analytical results agree well with accurate Mie theory. We also find that given the same parameters, a radially polarized Gaussian beam can hold a particle much nearer to the beam center than a linearly polarized Gaussian beam. We note that the radially polarized beam is known to be more effective as compared to the linearly polarized beam in the geometrical optics regime as well as in the Mie regime. Here we have shown that the radially polarized beam is also favorable in the Rayleigh regime, where the particles are much smaller than a wavelength.

In order to illustrate the superiority of the radially polarized Gaussian beam as compared to the linearly polarized Gaussian beam, we introduce a parameter

$$
R=F_{\text {grad }}\left(F_{a b s}+F_{\text {rad }}\right)+1 .
$$

$R<0$ implies that the particle can be trapped while $R>0$ implies that the particle cannot be trapped. In Fig. 4(a) we can see that a radially polarized Gaussian beam is capable of trapping particles with high dielectric constant, as compared with that of linearly polarization. Consider for example (we only present the analytical results because analytical and numerical results show no graphically discernible difference), a particle with $\varepsilon_{r}=5$ can be trapped by a radially polarized beam [see Fig. 4(c)], but not with a linearly polarized beam [see Fig. 4(b)]. We know that the effectiveness of optical trapping depends strongly on the ratio between the real and imaginary parts of $\varepsilon$. As $\varepsilon_{r}$ decreases, absorption dominates and thus $R$ increases rapidly, as shown in Fig. 4(a). On the other hand, for large $\varepsilon_{r}$, the absorption force can be neglected compared to the other forces, and the $R$ curves for
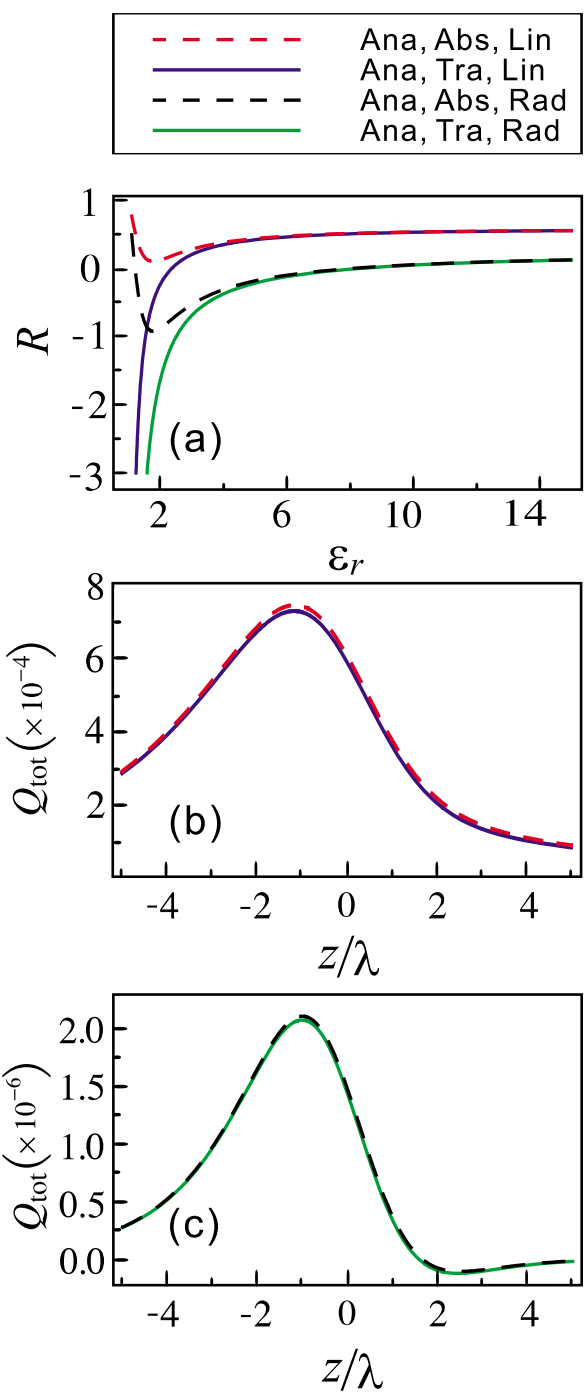

FIG. 4. (Color online) A spherical nanoparticle in air with $\rho$ $=0.5$, illuminated by a radially polarized or a linearly polarized Gaussian beam with the wavelength $\lambda=1064 \mathrm{~nm}$ and waist radius $w_{0}=\lambda$. Simplified analytical calculations for both the transparent particle and the absorptive particle $\left(\varepsilon_{i}=0.01\right)$ are considered. (a) Ratio $R$ versus $\varepsilon_{r}$, (b) and (c) are $Q_{\text {tot }}\left[=F_{\text {tot }} /(n P / c)\right]$ versus $z / \lambda$ for a linearly and a radially polarized Gaussian beam, respectively, with $\varepsilon_{r}=5, n$ the refractive index of the surrounding medium, $P$ the beam power, and $c$ the light velocity in vacuum. In the figure legend, Ana=Analytical, Abs=Absorption, Tra=Transparent, Lin $=$ Linear Polarization, Rad $=$ Radial Polarization.

the absorptive and transparent cases joint together. $R$ for other parameters are plotted in Fig. 5 for reference.

\section{CONCLUSION}

Using a mixed dipole model to describe the incident trapping beam, simple analytical expressions for equilibrium position, trap stiffness, and optical forces are derived using Mie theory in the long wavelength limit, which allows one to obtain qualitative information on optical trapping. The dependence of these quantities on dielectric constants and incident polarization are delineated. Analytical expressions of 


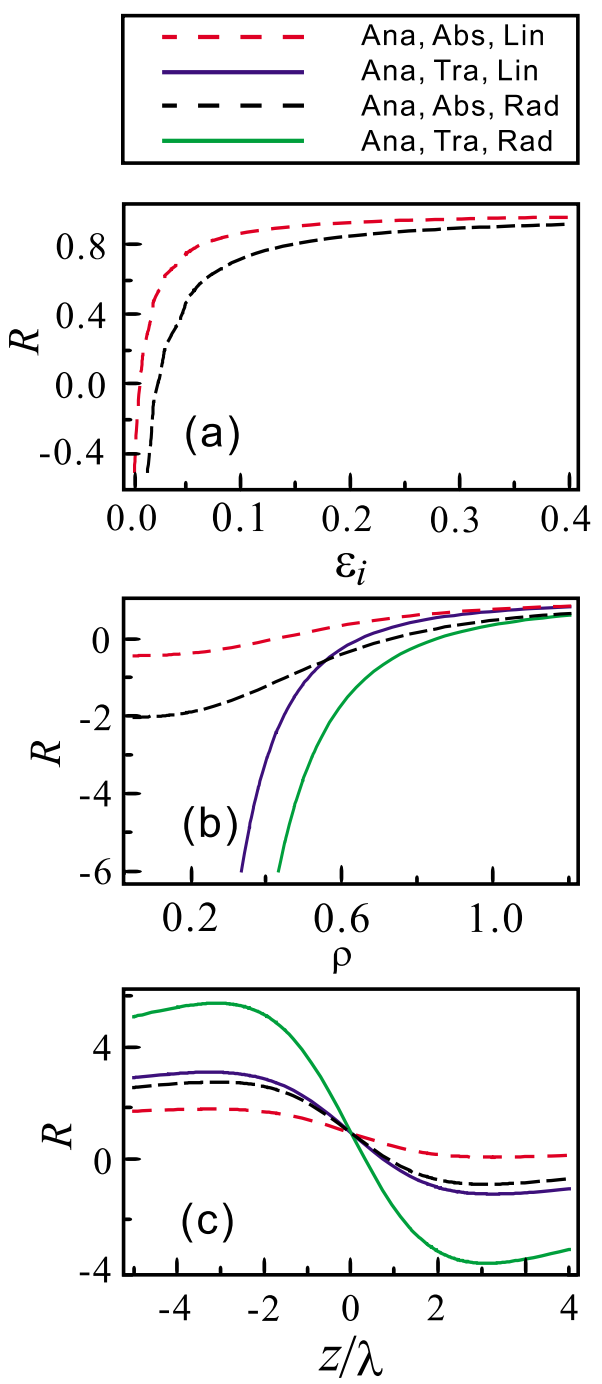

FIG. 5. (Color online) A spherical nanoparticle in air is illuminated by either a radially polarized or a linearly polarized Gaussian beam with the wavelength $\lambda=1064 \mathrm{~nm}$ and waist radius $w_{0}=\lambda$. Both the transparent particle $(\varepsilon=1.5)$ and the absorptive particle $(\varepsilon=1.5+0.01 i)$ are considered. (a) Ratio $R$ versus $\varepsilon_{i}$ with $\rho=0.5$, (b) Ratio $R$ versus $\rho$ and (c) Ratio $R$ versus $z / \lambda$ with $\rho=0.5$. In the figure legend, Ana=Analytical, Abs=Absorption, Tra $=$ Transparent, Lin $=$ Linear Polarization, Rad $=$ Radial Polarization .

forces are presented both for absorptive and transparent Rayleigh particles in a linearly or radially polarized Gaussian beam. By employing the mixed dipole model [23,25], our force expressions are results beyond paraxial approximation. Moreover, in the paraxial limit, our results correctly reduce to those of Harada. The equilibrium positions behave differently for absorptive and transparent particles. The $a^{3}$ and $w_{0}^{4}$ dependence of equilibrium positions is demonstrated for transparent Rayleigh particles, but for absorptive particles, the dependency is no longer $a^{3}$. The trap stiffness is found to be proportional to $a^{3}$ for both absorptive and transparent particles. Comparison between our analytical results and accurate numerical calculations are also presented. Finally, the superiority of radially polarized Gaussian beam is demonstrated. Our calculation is valid for a spherical particle. In the future work, it would be of interest to study the trapping forces acting on a nonspherical Rayleigh particle. We speculate that for a particle whose shape is close to a sphere, one may simply replace the $a^{3}$-term in Eq. (33), which is proportional to the volume of the particle, with the volume of the nonspherical particle (with appropriate prefactor).

\section{ACKNOWLEDGMENTS}

We thank W. K. Chen for discussion. This research is supported by NSFC, PCSIRT, and MOE of China (Grant No. B06011).

\section{APPENDIX A: SOME FACTORS IN FORCES EXPRESSIONS}

The factor $g_{0}$ in Eq. (22) is given by

$$
\begin{aligned}
g_{0}= & \frac{1}{1+\eta^{2}}-\frac{3}{\vartheta} \frac{1}{\left(1+\eta^{2}\right)^{2}}+\frac{5}{\vartheta^{2}} \frac{1-\eta^{2} / 5}{\left(1+\eta^{2}\right)^{3}} \\
& -\left[\frac{5-\eta^{2}}{\vartheta^{3}}+\frac{11-\eta^{2}}{4 \vartheta^{4}}-\frac{3}{4 \vartheta^{5}}\right]\left(1+\eta^{2}\right)^{-4} .
\end{aligned}
$$

where $\eta=\varrho / \vartheta$.

The factor $h_{0}$ and $h_{1}$ involved in the gradient force expression Eq. (26) for a transparent particle illuminated by a linearly polarized Gaussian beam are given by

$$
\begin{aligned}
h_{0}= & \left(6 \vartheta^{-2}-3 \vartheta^{-3}+3 \vartheta^{-4} / 4\right)\left(1+\eta^{2}\right)^{-4} \\
& -4 \vartheta^{-1}\left(1+\eta^{2}\right)^{-3}+\left(1+\eta^{2}\right)^{-2}, \\
h_{1}= & \left(1+\eta^{2}\right)^{-5}\left\{(360-1080 \vartheta)(\varepsilon-1)(\varepsilon+2)^{2}\right. \\
- & 24 \vartheta^{4}\left(42+49 \varepsilon-45 \varepsilon^{2}-45 \varepsilon^{3}-\varepsilon^{4}\right)\left(1+\eta^{2}\right) \\
- & 16 \vartheta^{5}\left(18-49 \varepsilon+30 \varepsilon^{3}+\varepsilon^{4}\right)\left(1+\eta^{2}\right)^{2} \\
+ & 4 \vartheta^{6}(\varepsilon-1)^{2}\left(38+27 \varepsilon+\varepsilon^{2}\right)\left(1+\eta^{2}\right)^{3} \\
- & 12 \vartheta^{3}(\varepsilon-1)\left[362+42 \eta^{2}+\varepsilon^{3}\left(1+\eta^{2}\right)\right. \\
+ & \left.2 \varepsilon^{2}\left(63+23 \eta^{2}\right)+\varepsilon\left(411+91 \eta^{2}\right)\right] \\
+ & 3 \vartheta^{2}(\varepsilon-1)\left[\varepsilon^{3}\left(1+\eta^{2}\right)+6\left(327+7 \eta^{2}\right)\right. \\
+ & \left.\left.\varepsilon^{2}\left(526+46 \eta^{2}\right)+\varepsilon\left(2011+91 \eta^{2}\right)\right]\right\},
\end{aligned}
$$

The factor $q\left(n_{R}, n_{I}\right), p\left(n_{R}, n_{I}\right), u\left(n_{R}, n_{I}\right)$, and $v\left(n_{R}, n_{I}\right)$ involved in the force expressions of Eqs. (34) and (39) for an absorptive particle are given by

$$
\begin{aligned}
q\left(n_{R}, n_{I}\right)= & {\left[\left(n_{R}^{2}+n_{I}^{2}\right)^{2}+4\left(n_{R}^{2}-n_{I}^{2}+1\right)\right]^{-2} } \\
& \times\left[\left(2-n_{R}^{2}-n_{R}^{4}\right)^{2}-2\left(1-2 n_{R}^{2}\right) n_{I}^{6}+2\left(2-23 n_{R}^{2}\right.\right. \\
& \left.\left.+n_{R}^{4}+2 n_{R}^{6}\right) n_{I}^{2}+n_{I}^{8}-\left(3+2 n_{R}^{2}-6 n_{R}^{4}\right)\right], \quad(\mathrm{A} 4) \\
p\left(n_{R}, n_{I}\right)= & n_{R} n_{I}\left\{\frac{126}{\left(n_{R}^{2}+n_{I}^{2}\right)^{2}+4\left(n_{R}^{2}-n_{I}^{2}+1\right)}\right. \\
& +\frac{432\left(n_{I}^{2}-n_{R}^{2}-2\right)}{\left[\left(n_{R}^{2}+n_{I}^{2}\right)^{2}+4\left(n_{R}^{2}-n_{I}^{2}+1\right)\right]^{2}}+1 \\
& \left.+\frac{25}{4\left(n_{R}^{2}+n_{I}^{2}\right)^{2}+3\left(4 n_{R}^{2}-4 n_{I}^{2}+3\right)}\right\}, \quad \text { (A5) }
\end{aligned}
$$




$$
\begin{aligned}
& u\left(n_{R}, n_{I}\right)=\frac{3 n_{R} n_{I}}{\left(n_{R}^{2}+n_{I}^{2}\right)^{2}+4\left(n_{R}^{2}-n_{I}^{2}+1\right)}, \\
& v\left(n_{R}, n_{I}\right)=\frac{\left(n_{R}^{2}+n_{I}^{2}\right)^{2}+n_{R}^{2}-n_{I}^{2}-2}{\left(n_{R}^{2}+n_{I}^{2}\right)^{2}+4\left(n_{R}^{2}-n_{I}^{2}+1\right)} .
\end{aligned}
$$

\section{APPENDIX B: DIFFERENCES BETWEEN OUR RESULTS AND THOSE BY HARADA et al.}

In this appendix, we tabulate the differences between our analytical force expressions and those by Harada and Asakura [8].

$$
F_{z, s c a}^{o u r}-F_{z, s c a}^{H K}=-\frac{\pi \varepsilon_{0} E_{0}^{2}}{3 k^{2}} \frac{(\varepsilon-1)^{2}}{(\varepsilon+2)^{2}} d(\vartheta, \varrho) \rho^{6},
$$

$$
\begin{aligned}
F_{z, \text { grad }}^{\text {our }}-F_{z, \text { grad }}^{H K}= & -\frac{\pi \varepsilon_{0} E_{0}^{2}}{2 k^{2}} \frac{(\varepsilon-1)}{(\varepsilon+2)} \frac{\varrho \vartheta^{2} \rho^{3}}{\left(\vartheta^{2}+\varrho^{2}\right)^{4}} \\
& \times\left[3-16 \vartheta^{3}+24 \vartheta^{2}-4 \vartheta\left(3+4 \varrho^{2}\right)\right],
\end{aligned}
$$

with

$$
\begin{aligned}
d(\vartheta, \varrho)= & \frac{\vartheta^{2}}{\left(\vartheta^{2}+\varrho^{2}\right)^{4}}\left[\left(\varrho^{2}+4 \varrho^{4}\right)+12 \vartheta^{5}+4 \vartheta^{3}\left(5+6 \varrho^{2}\right)\right. \\
& \left.-\vartheta^{2}\left(11+16 \varrho^{2}\right)-20 \vartheta^{4}+\vartheta\left(3-4 \varrho^{2}+12 \varrho^{4}\right)\right]
\end{aligned}
$$

When $\vartheta \rightarrow \infty$, the difference between Eq. (B1) and (B2) vanish, and the two approaches become equivalent.
[1] A. Ashkin, J. M. Dziedzic, J. E. Bjorkholm, and S. Chu, Opt. Lett. 11, 288 (1986).

[2] A. Ashkin, Phys. Rev. Lett. 24, 156 (1970).

[3] A. Ashkin, Science 210, 1081 (1980).

[4] A. Ashkin and J. M. Dziedzic, Science 235, 1517 (1987).

[5] A. Ashkin, J. M. Dziedzic, and T. Yamane, Nature (London) 330, 769 (1987).

[6] A. Ashkin and J. M. Dziedzic, Proc. Natl. Acad. Sci. U.S.A. 86, 7914 (1989).

[7] F. Xu, K. F. Ren, G. Gouesbet, X. S. Cai, and G. Gréhan, Phys. Rev. E 75, 026613 (2007).

[8] Y. Harada and T. Asakura, Opt. Commun. 124, 529 (1996).

[9] Y. K. Nahmias and D. J. Oddl, IEEE J. Quantum Electron. 38, 131 (2002).

[10] J. Barton, D. Alexander, and S. Schaub, J. Appl. Phys. 66, 4594 (1989).

[11] R. Gussgard, T. Lindmo, and I. Brevik, J. Opt. Soc. Am. B 9, 1922 (1992).

[12] J. S. Kim and S. S. Lee, J. Opt. Soc. Am. 73, 303 (1983).

[13] A. Rohrbach and E. H. K. Stelzer, Appl. Opt. 41, 2494 (2002).

[14] J. A. Lock, S. Y. Wrbanek, and K. E. Weiland, Appl. Opt. 45, 3634 (2006).

[15] D. Ganic, X. Gan, and M. Gu, Opt. Express 12, 2670 (2004).

[16] D. G. Grier, Nature (London) 424, 810 (2003).

[17] A. Rohrbach, Phys. Rev. Lett. 95, 168102 (2005).

[18] N. B. Viana, A. Mazolli, P. A. Maia Neto, H. M. Nussenzveig, M. S. Rocha, and O. N. Mesquita, Appl. Phys. Lett. 88, 131110 (2006).

[19] M. J. Lang and S. M. Block, Am. J. Phys. 71, 201 (2003).

[20] K. C. Neuman and S. M. Block, Rev. Sci. Instrum. 75, 2787 (2004).

[21] Q. Zhan, Opt. Express 12, 3377 (2004).

[22] A. Ashkin, Biophys. J. 61, 569 (1992).

[23] C. J. R. Sheppard and S. Saghafi, J. Opt. Soc. Am. A Opt. Image Sci. Vis 16, 1381 (1999).

[24] J. F. Nye and M. Berry, Proc. R. Soc. London, Ser. A 336, 165 (1974).

[25] C. J. R. Sheppard and S. Saghafi, Opt. Lett. 24, 1543 (1999).
[26] P. M. Hansen, V. K. Bhatia, N. Harrit, and L. Oddershede, Nano Lett. 5, 1937 (2005).

[27] H. Kawauchi, K. Yonezawa, Y. Kozawa, and S. Sato, Opt. Lett. 32, 1839 (2007)

[28] T. A. Nieminen, N. R. Heckenberg, and H. RubinszteinDunlop, Opt. Lett. 33, 122 (2008).

[29] J. Ng, Z. F. Lin, C. T. Chan, and P. Sheng, Phys. Rev. B 72, 085130 (2005).

[30] J. Ng, C. T. Chan, P. Sheng, and Z. F. Lin, Opt. Lett. 30, 1956 (2005).

[31] J. Ng and C. T. Chan, Appl. Phys. Lett. 92, 251109 (2008).

[32] J. Ng, R. Tang, and C. T. Chan, Phys. Rev. B 77, 195407 (2008).

[33] Y. L. Xu, Appl. Opt. 34, 4573 (1995).

[34] C. F. Bohren and D. R. Huffman, Absorption and Scattering of Light by Small Particles (John Wiley and Sons, New York, 1983).

[35] M. I. Mishchenko, L. D. Travis, and A. A. Lacis, Scattering, Absorption and Emission of Light by Small Particles (Cambridge University Press, Cambridge, 2002).

[36] M. J. Berg, A. Chakrabarti, and C. M. Sorensen, J. Quant. Spectrosc. Radiat. Transf. 110, 43 (2009).

[37] M. I. Mishchenko, M. J. Berg, C. M. Sorensen, and C. V. M. van der Mee, J. Quant. Spectrosc. Radiat. Transf. 110, 323 (2009)

[38] L. D. Laudau and Ye. M. Lifshitz, Brief Course in Theoretical Physics. Mechanics. Electrodynamics Book 1 (Nauka, Moscow, 1969).

[39] V. V. Kotlyar and A. G. Nalimov, J. Mod. Opt. 53, 1829 (2006).

[40] A. Mazolli, P. A. Maia Neto, and H. M. Nussenzveig, Proc. R. Soc. London, Ser. A 459, 3021 (2003).

[41] R. Pobre and C. Saloma, Appl. Opt. 41, 7694 (2002).

[42] S. R. Seshadri, Opt. Lett. 32, 3218 (2007).

[43] A. April, Opt. Lett. 33, 1563 (2008).

[44] J. D. Jackson, Classical electrodynamics, 3rd ed. (Wiley, New York, 1999). 